\title{
Development of a Fluorescent-Labeled Trapping Reagent to Detect Reactive
}

\section{Acyl Glucuronides}

Chikako Shibazaki ${ }^{\dagger}$, Okishi Mashita $^{\ddagger}$, Kyoko Takahashi ${ }^{\S}$, Shigeo Nakamura ${ }^{\S}$ Tadahiko Mashino ${ }^{\dagger}$, Tomoyuki Ohe ${ }^{\dagger, *}$

${ }^{\dagger}$ Faculty of Pharmacy, Keio University, 1-5-30 Shibakoen, Minato-ku, Tokyo, Japan

‡ Laboratory for Safety Assessment and ADME, Pharmaceuticals Research Center, Asahi Kasei Pharma Corporation, 632-1 Mifuku, Izunokuni, Shizuoka, Japan

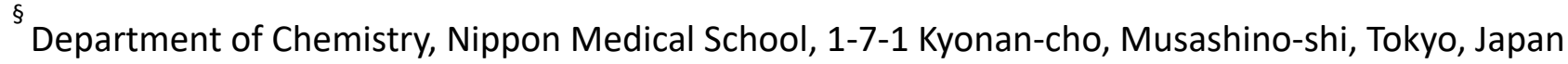

*Corresponding Author: Tomoyuki Ohe, Ph.D., Phone: +81 35400 2691, Fax: +81 35400 2691, Email: ohe-tm@pha.keio.ac.jp

Chemicals and Materials.

Figure $\mathbf{S 1} .{ }^{1} \mathrm{H}$ NMR spectrum of Dap-Dan.

Figure S2. ${ }^{13} \mathrm{C}$ NMR spectrum of Dap-Dan.

Synthesis of $\beta$ Ala-Dan.

Figure S3. ${ }^{1} \mathrm{H}$ NMR spectrum of $\beta$ Ala-Dan.

Figure S4. ${ }^{13} \mathrm{C}$ NMR spectrum of $\beta$ Ala-Dan.

Synthesis of dKF.

Synthesis of ACL1.

Figure S5. ${ }^{1} \mathrm{H}$ NMR spectrum of ACL1. 
Figure S6. ${ }^{13} \mathrm{C}$ NMR spectrum of ACL1.

Figure S7. ${ }^{1} \mathrm{H}-{ }^{1} \mathrm{H}$ COSY spectrum of ACL1.

Synthesis of ACL2.

Figure S8. ${ }^{1} \mathrm{H}$ NMR spectrum of ACL2.

Figure S9. ${ }^{13} \mathrm{C}$ NMR spectrum of $\mathrm{ACL} 2$.

Figure S10. ${ }^{1} \mathrm{H}-{ }^{1} \mathrm{H}$ COSY spectrum of $\mathrm{ACL} 2$.

Quantitative Analysis of Adduct Formation.

Figure S11. Correlation between the \% of B and reciprocal of corrected constant $(f)$ for Dap-Dan, $\beta A l a-D a n$ and dKF.

Table S1. Retention time for each adduct.

Chemicals and Materials.

Boc-Dap(Boc)-OH, Boc- $\beta-A l a-O H$, Boc-Lys(Boc)-OH, H-Phe-OBzl, Boc-Dap-OH and H-Dap(Boc)-OH were purchased from Watanabe Chemical Industries, Ltd. (Hiroshima, Japan). Ethylenediamine, 1,5diaminopentane, dansyl chloride, 1-(3-dimethylaminopropyl)-3-ethylcarbodiimide, 1hydroxybenzotriazole monohydrate, diclofenac, indomethacin, ketoprofen, fenoprofen calcium salt dihydrate, mefenamic acid, probenecid, furosemide, repaglinide, gemfibrozil and montelukast were purchased from Tokyo Chemical Industry Co., Ltd. (Tokyo, Japan). Methanol and acetonitrile were purchased from Kanto Chemical Co., Inc. (Tokyo, Japan). Tolmetin was purchased from LKT Laboratories, Inc. (Minnesota, USA). $1 \mathrm{M}$ Sodium hydroxide solution, $1 \mathrm{M}$ hydrochloric acid and 
ibuprofen were purchased from FUJIFILM Wako Pure Chemical Corporation (Osaka, Japan). Phosphate buffer powder, dimethyl sulfoxide (DMSO), zomepirac sodium salt, bromfenac sodium and ibufenac were purchased from Sigma-Aldrich Co. LCC (St. Louis, USA). Benoxaprofen and fenclofenac were purchased from Toronto Research Chemicals Inc. (Toronto, Canada). Hyper-grade acetonitrile for LC/MS was purchased from MilliporeSigma (Massachusetts, USA).

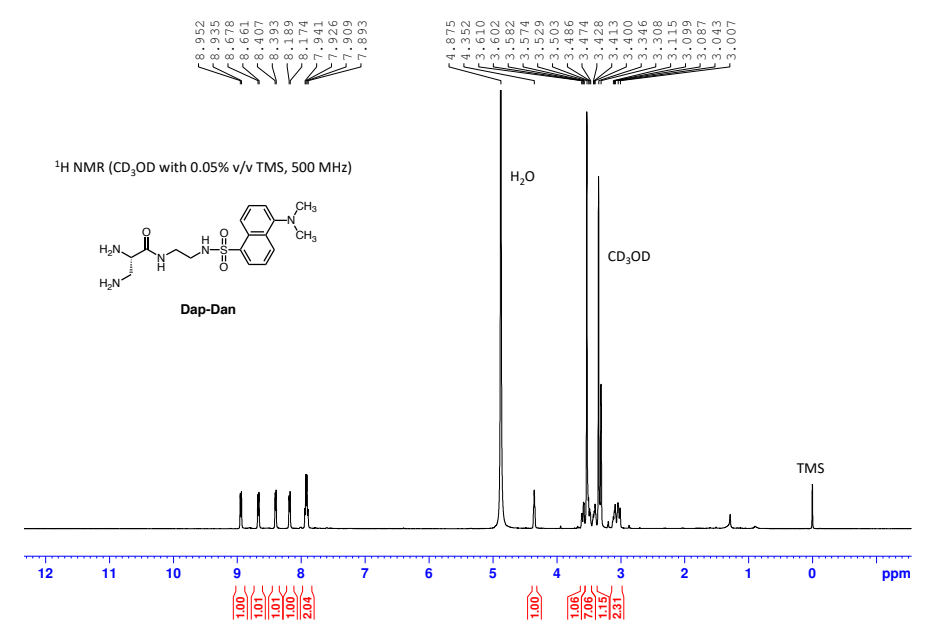

Figure S1. ${ }^{1} \mathrm{H}$ NMR spectrum of Dap-Dan.

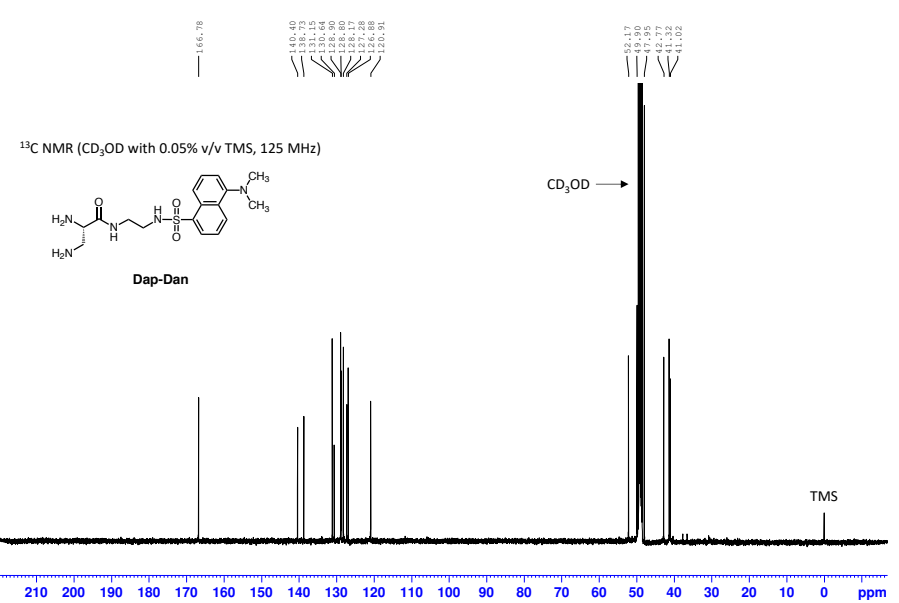

Figure S2. ${ }^{13} \mathrm{C}$ NMR spectrum of Dap-Dan. 


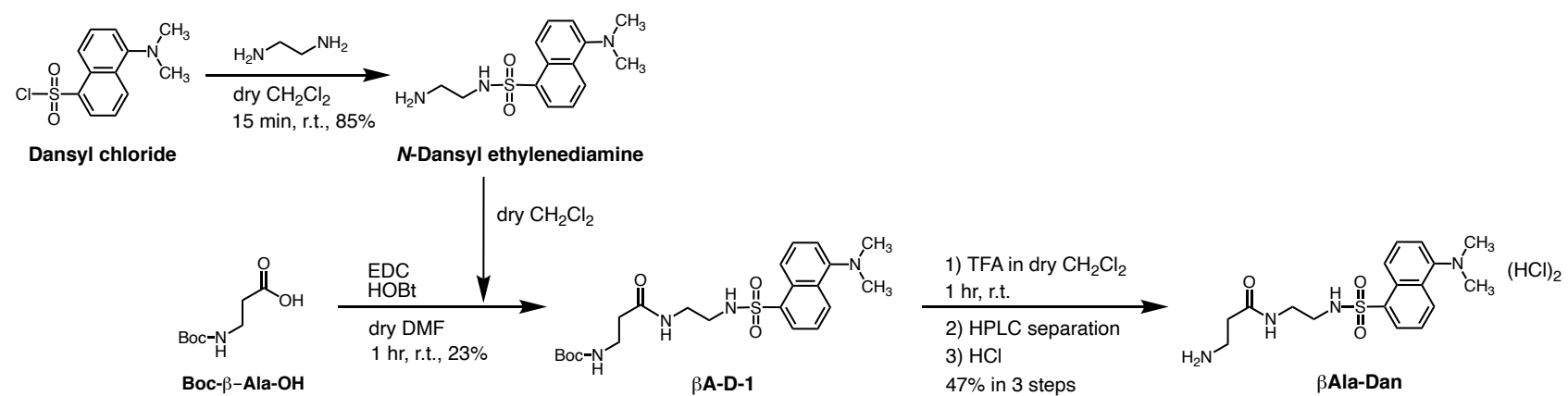

Scheme S1. Synthetic route for $\beta$ Ala-Dan.

Synthesis of $\beta$ Ala-Dan. Synthesis of $\mathbf{N}$-Dansyl ethylenediamine. Dansyl chloride (100 mg, 0.372 mmol) was dissolved in $5.0 \mathrm{~mL}$ of dry dichloromethane. Ethylenediamine (112 mg, $124 \mu \mathrm{L}, 1.86$ mmol) was added and then the mixture was stirred for $30 \mathrm{~min}$. Chloroform and saturated sodium hydrogen carbonate solution were added and the organic layer was separated. The water layer was extracted with chloroform. The organic layers were combined, washed with brine, dried over anhydrous sodium sulfate, and evaporated under high vacuum. The resulting residue was purified by column chromatography $\left(\mathrm{CHCl}_{3}: \mathrm{MeOH}=10: 1\right)$, affording $92.9 \mathrm{mg}$ of yellow solid (85\% yield). Synthesis of $\beta A-D-1$. Boc- $\beta$-Ala-OH $(29.9 \mathrm{mg}, 0.158 \mathrm{mmol})$ was dissolved in $1.0 \mathrm{~mL}$ of dry dichloromethane. 1-(3-Dimethylaminopropyl)-3-ethylcarbodiimide $(45.5 \mathrm{mg}, 0.237 \mathrm{mmol})$ and 1hydroxybenzotriazole monohydrate $(32.0 \mathrm{mg}, 0.237 \mathrm{mmol})$ suspended in $1.0 \mathrm{~mL}$ of dry DMF was added and the mixture was stirred for $30 \mathrm{~min}$, then $\mathrm{N}$-dansyl ethylenediamine $(39.4 \mathrm{mg}, 0.134$ $\mathrm{mmol}$ ) dissolved in $2.0 \mathrm{~mL}$ of dry dichloromethane was added. The mixture was kept stirred for another $30 \mathrm{~min}$. Chloroform and $1 \mathrm{M}$ hydrochloric acid were added and the organic layer was separated. The water layer was extracted with chloroform and the organic layers were combined, 
washed with brine, dried over anhydrous sodium sulfate, and evaporated under high vacuum. The resulting residue was purified by column chromatography $\left(\mathrm{CH}_{2} \mathrm{Cl}_{2}: \mathrm{MeOH}=100: 1 \rightarrow 10: 1\right)$, affording $14.2 \mathrm{mg}$ of yellow paste (23\% yield). $\beta$ A-D-1: ${ }^{1} \mathrm{H}$ NMR $\left(\mathrm{CDCl}_{3}\right.$ with $\left.0.03 \% \mathrm{v} / \mathrm{v} \mathrm{TMS}, 500 \mathrm{MHz}\right): \delta_{\mathrm{H}} 1.41$ $\left(\mathrm{s},-\mathrm{C}\left(\mathrm{CH}_{3}\right)_{3}, 9 \mathrm{H}\right), 2.22\left(\mathrm{t}, \mathrm{J}=5.6 \mathrm{~Hz},-\mathrm{CH}_{2^{-}}, 2 \mathrm{H}\right), 2.89\left(\mathrm{~s},-\mathrm{N}\left(\mathrm{CH}_{3}\right)_{2}, 6 \mathrm{H}\right), 3.02-3.05\left(\mathrm{~m},-\mathrm{CH}_{2^{-}}, 2 \mathrm{H}\right), 3.28-$ $3.33\left(\mathrm{~m},-\mathrm{CH}_{2^{-}},-\mathrm{CH}_{2^{-}}, 4 \mathrm{H}\right), 5.16(\mathrm{br},-\mathrm{NH}-, 1 \mathrm{H}), 6.00(\mathrm{br},-\mathrm{NH}-, 1 \mathrm{H}), 6.34(\mathrm{br},-\mathrm{NH}-, 1 \mathrm{H}), 7.18(\mathrm{~d}, \mathrm{~J}=7.5$ $\mathrm{Hz}, \operatorname{Ar} H, 1 \mathrm{H}), 7.52(\mathrm{t}, J=7.9 \mathrm{~Hz}, \operatorname{Ar~H}, 1 \mathrm{H}), 7.56(\mathrm{t}, J=8.1 \mathrm{~Hz}, \operatorname{ArH}, 1 \mathrm{H}), 8.22(\mathrm{~d}, J=7.2 \mathrm{~Hz}, \operatorname{Ar} H, 1 \mathrm{H})$, 8.29 (d, J=8.6 Hz, Ar H, 1H), 8.54 (d, J=8.5 Hz, Ar H, 1H). Synthesis of BAla-Dan. BA-D-1 (14.2 mg, $0.0306 \mathrm{mmol}$ ) was dissolved in $500 \mu \mathrm{L}$ of dry dichloromethane, and trifluoroacetic acid (105 mg, $70.2 \mu \mathrm{L}, 0.918 \mathrm{mmol}$ ) was added. After stirring for $60 \mathrm{~min}$, the solvent was evaporated under vacuum. The resulting residue was purified by a high-performance liquid chromatography (HPLC, 1260, Agilent Technologies, Inc., California, USA) equipped with InertSustain C18 5 $\mu$ M 20×150 mm (GL Sciences, Tokyo, Japan). The mobile phases were water containing $0.1 \%$ formic acid (A) and acetonitrile containing $0.1 \%$ formic acid (B), and the flow rate was set at $3.0 \mathrm{~mL} / \mathrm{min}$. The profile of the gradient is as follows: $20 \%$ B for $3 \mathrm{~min}, 20-69 \%$ B in $17 \mathrm{~min}, 69 \%$ B for $10 \mathrm{~min}, 69-20 \% \mathrm{~B}$ in 0.1 min followed by $20 \%$ B for another $9.9 \mathrm{~min}$ ( $40 \mathrm{~min}$ in total). The earned paste was washed with 1 $\mathrm{M}$ hydrochloric acid and dried under vacuum, affording $6.3 \mathrm{mg}$ of a white amorphous solid (47\% yield, $>99.5 \%$ pure as assessed by HPLC/UV). $\beta$ Ala-Dan: ${ }^{1} \mathrm{H}$ NMR $\left(\mathrm{D}_{2} \mathrm{O}\right.$ with $\left.0.05 \% \mathrm{v} / \mathrm{v} \mathrm{TMS}, 600 \mathrm{MHz}\right)$ : $\delta_{\mathrm{H}}$ 2.52-2.55 (m, $\left.-\mathrm{CH}_{2^{-}}, 2 \mathrm{H}\right), 3.12-3.15\left(\mathrm{~m},-\mathrm{CH}_{2^{-}}, 2 \mathrm{H}\right), 3.20-3.25\left(\mathrm{~m},-\mathrm{CH}_{2^{-}},-\mathrm{CH}_{2^{-}}, 4 \mathrm{H}\right), 3.54\left(\mathrm{~s},-\mathrm{N}\left(\mathrm{CH}_{3}\right)_{2}\right.$, $6 H)$, 7.94-7.97 (m, $\operatorname{Ar~H,~2H),~8.12-8.13~(m,~} \operatorname{Ar~H,1H),~8.40-8.42~(m,~Ar~H,~1H),~8.47-8.49~(m,~Ar~H,~}$ 1H), 8.78-8.80 (m, Ar H, 1H). ${ }^{13} \mathrm{C} N M R\left(D_{2} \mathrm{O}, 150 \mathrm{MHz}\right): \delta c 31.9,35.6,39.0,41.5,46.7$ (2C), 119.4, 
125.70, 126.72, 126.5, 127.0, 128.1, 128.6, 130.2, 135.4, 138.6, 172.2. HRMS-ESI $(\mathrm{m} / \mathrm{z}):[\mathrm{M}+\mathrm{H}]^{+}{ }^{+}$calcd for $\mathrm{C}_{17} \mathrm{H}_{25} \mathrm{~N}_{4} \mathrm{O}_{3} \mathrm{~S}, 365.1647$; found, 365.1618 (-2.9 mmu).

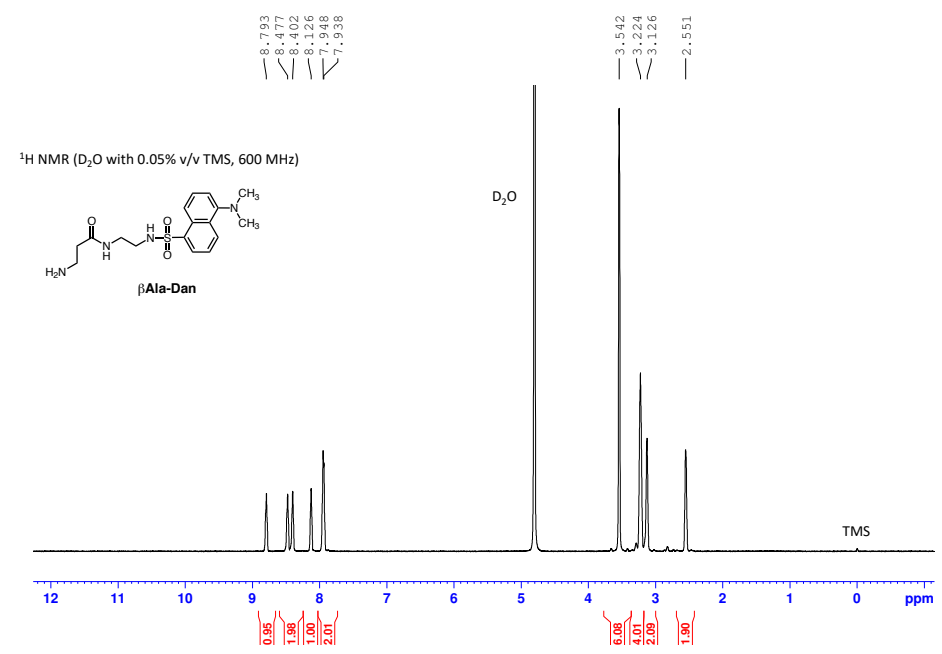

Figure S3. ${ }^{1} \mathrm{H}$ NMR spectrum of $\beta$ Ala-Dan.

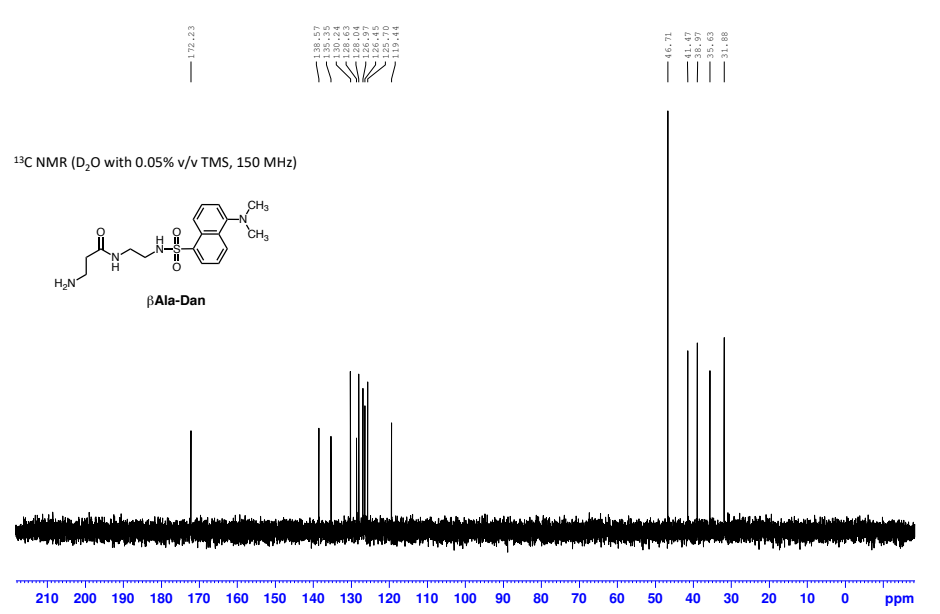

Figure S4. ${ }^{13} \mathrm{C}$ NMR spectrum of $\beta$ Ala-Dan. 


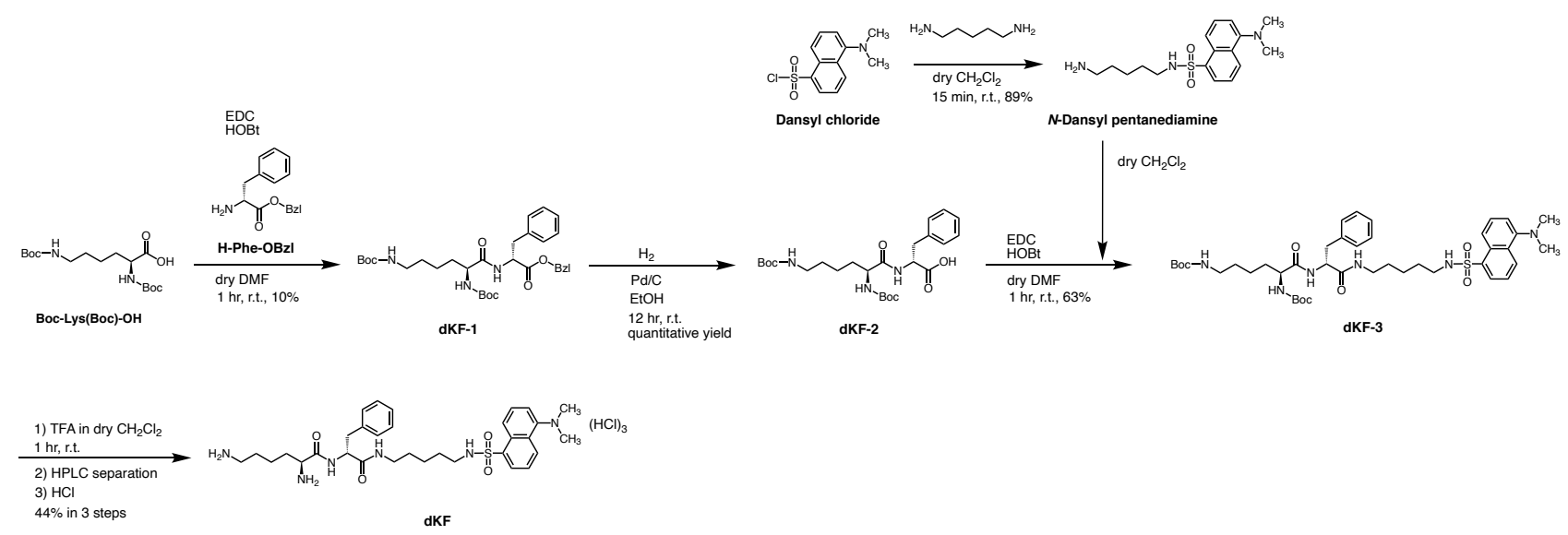

Scheme S2. Synthetic route for dKF.

Synthesis of dKF. Synthesis of dKF-1. Boc-(Lys)Boc-OH (107 mg, $0.309 \mathrm{mmol}$ ) was dissolved in 2.0 $\mathrm{mL}$ of dry DMF. 1-(3-Dimethylaminopropyl)-3-ethylcarbodiimide (72.6 mg, $0.378 \mathrm{mmol}$ ) and 1hydroxybenzotriazole monohydrate $(52.6 \mathrm{mg}, 0.347 \mathrm{mmol})$ suspended in $2.0 \mathrm{~mL}$ of dry DMF was added and the mixture was stirred for $30 \mathrm{~min}$, then $\mathrm{H}-\mathrm{Phe}-\mathrm{OBzl}(45.6 \mathrm{mg}, 0.57 \mathrm{mmol})$ dissolved in $2.0 \mathrm{~mL}$ of dry dichloromethane was added. The mixture was kept stirred for another $15 \mathrm{~min}$. Ethyl acetate and $1 \mathrm{M}$ hydrochloric acid were added and the organic layer was separated. The water layer was extracted with ethyl acetate and the organic layers were combined, washed with brine, dried over anhydrous sodium sulfate, and evaporated under high vacuum. The resulting residue was purified by column chromatography $\left(\mathrm{CH}_{2} \mathrm{Cl}_{2}: \mathrm{MeOH}=100: 1 \rightarrow 90: 1 \rightarrow 75: 1\right)$, affording $17.3 \mathrm{mg}$ of colorless and transparent paste (10\% yield). dKF-1: ${ }^{1} \mathrm{H} \mathrm{NMR}\left(\mathrm{CDCl}_{3}\right.$ with $\left.0.03 \% \mathrm{v} / \mathrm{v} \mathrm{TMS}, 500 \mathrm{MHz}\right)$ : $\delta_{\mathrm{H}}$ 1.26-1.34 (m, $\left.-\mathrm{CH}_{2^{-}}, 2 \mathrm{H}\right), 1.43\left(\mathrm{~s},-\mathrm{C}\left(\mathrm{CH}_{3}\right)_{3}, 9 \mathrm{H}\right), 1.44\left(\mathrm{~s},-\mathrm{C}\left(\mathrm{CH}_{3}\right)_{3}, 9 \mathrm{H}\right), 1.45-1.49\left(\mathrm{~m},-\mathrm{CH}_{2^{-}}, 2 \mathrm{H}\right)$, 1.52-1.56 (m, $\left.-\mathrm{CH}_{2^{-}}, 1 \mathrm{H}\right), 1.75-1.79\left(\mathrm{~m},-\mathrm{CH}_{2^{-}}, 1 \mathrm{H}\right), 3.05-3.09\left(\mathrm{~m},-\mathrm{CH}_{2^{-}}, 2 \mathrm{H}\right), 3.11-3.16\left(\mathrm{~m},-\mathrm{CH}_{2^{-}}, 2 \mathrm{H}\right)$, 
4.00-4.03 (m, -CH-, 1H), 4.62 (s, -NH-, 1H), 4.89 (dd, J=6.4, $13.1 \mathrm{~Hz},-\mathrm{CH}-, 1 \mathrm{H}), 5.07$ (br, -NH-, 1H), $5.10\left(\mathrm{~d}, \mathrm{~J}=12.2 \mathrm{~Hz},-\mathrm{CH}_{2^{-}}, 1 \mathrm{H}\right), 5.16\left(\mathrm{~d}, \mathrm{~J}=12.1 \mathrm{~Hz},-\mathrm{CH}_{2^{-}}, 1 \mathrm{H}\right), 6.48$ (d, J=6.7 Hz, $\left.-\mathrm{NH}-, 1 \mathrm{H}\right), 7.01-7.03$ (m, Ar H, 2H), 7.21-7.22 (m, Ar H, 3H), 7.28-7.29 (m, Ar H, 2H), 7.35-7.37 (m, Ar H, 3H). Synthesis of dKF-2. dKF-1 (17.0 mg, $0.0292 \mathrm{mmol})$ was dissolved in $5.0 \mathrm{~mL}$ of ethanol, and $10 \% \mathrm{Pd} / \mathrm{C}(1.7 \mathrm{mg})$ was added. The reaction mixture was stirred for $12 \mathrm{hr}$ in a hydrogen atmosphere; and then, the mixture was filtered through celite. The filtrate was evaporated in vacuum, affording $15.2 \mathrm{mg}$ of colorless and transparent paste (quantitative yield). dKF-2: ${ }^{1} \mathrm{H} \mathrm{NMR}\left(\mathrm{CDCl}_{3}\right.$ with $0.03 \% \mathrm{v} / \mathrm{v} \mathrm{TMS}, 500$ $\mathrm{MHz}): \delta_{\mathrm{H}} 1.18-1.25\left(\mathrm{~m},-\mathrm{CH}_{2^{-}}, 2 \mathrm{H}\right), 1.40\left(\mathrm{~s},-\mathrm{C}\left(\mathrm{CH}_{3}\right)_{3}, 9 \mathrm{H}\right), 1.42\left(\mathrm{~s},-\mathrm{C}\left(\mathrm{CH}_{3}\right)_{3}, 9 \mathrm{H}\right), 1.45-1.50\left(\mathrm{~m},-\mathrm{CH}_{2^{-}}\right.$, $2 \mathrm{H}), 1.52-1.55\left(\mathrm{~m},-\mathrm{CH}_{2^{-}}, 1 \mathrm{H}\right), 1.64-1.67\left(\mathrm{~m},-\mathrm{CH}_{2^{-}}, 1 \mathrm{H}\right), 2.96-3.00\left(\mathrm{~m},-\mathrm{CH}_{2^{-}}, 2 \mathrm{H}\right), 3.02-3.05\left(\mathrm{~m},-\mathrm{CH}_{2^{-}}\right.$ , $1 \mathrm{H}), 3.18-3.22\left(\mathrm{~m},-\mathrm{CH}_{2^{-}}, 1 \mathrm{H}\right), 4.00-4.03(\mathrm{~m},-\mathrm{CH}-, 1 \mathrm{H}), 4.66(\mathrm{br},-\mathrm{NH}-, 1 \mathrm{H}), 4.81-4.85(\mathrm{~m},-\mathrm{CH}-, 1 \mathrm{H})$, 5.47 (br, -NH-, 1H), 6.88 (br, -NH-, 1H), 7.17- 7.20 (m, Ar H, 5H). Synthesis of N-Dansyl pentanediamine. Dansyl chloride $(15.0 \mathrm{mg}, 0.0558 \mathrm{mmol})$ was dissolved in $2.0 \mathrm{~mL}$ of dry dichloromethane. 1,5-diamino pentane $(28.5 \mathrm{mg}, 33.0 \mu \mathrm{L}, 0.279 \mathrm{mmol})$ was added and then the mixture was stirred for $15 \mathrm{~min}$. Dichloromethane and saturated sodium hydrogen carbonate solution were added and the organic layer was separated. The water layer was extracted with dichloromethane. The organic layers were combined, washed with brine, dried over anhydrous sodium sulfate, and evaporated under high vacuum. The resulting residue was purified by column chromatography $\left(\mathrm{CH}_{2} \mathrm{Cl}_{2}: \mathrm{MeOH}=75: 1 \rightarrow 30: 1 \rightarrow 15: 1\right)$, affording $16.7 \mathrm{mg}$ of yellow solid (89\% yield). N-Dansyl pentanediamine: ${ }^{1} \mathrm{H}$ NMR $\left(\mathrm{CDCl}_{3}\right.$ with $\left.0.03 \% \mathrm{v} / \mathrm{v} \mathrm{TMS}, 500 \mathrm{MHz}\right): \delta_{\mathrm{H}} 1.71-1.21\left(\mathrm{~m},-\mathrm{CH}_{2^{-}}\right.$, $2 \mathrm{H}), 1.23-1.27\left(\mathrm{~m},-\mathrm{CH}_{2^{-}}, 2 \mathrm{H}\right), 2.53\left(\mathrm{t}, \mathrm{J}=7.5 \mathrm{~Hz},-\mathrm{CH}_{2^{-}}, 2 \mathrm{H}\right), 2.88-2.89\left(\mathrm{~m},-\mathrm{CH}_{2^{-}}, 2 \mathrm{H}\right), 2.89\left(\mathrm{~s},-\mathrm{N}\left(\mathrm{CH}_{3}\right)_{2}\right.$, 
6H), 7.19 (d, J=7.4 Hz, Ar H, 1H), 7.51 (t, J=7.9 Hz, Ar H, 1H), 7.56 (t, J=8.0 Hz, Ar H, 1H), 8.25 (d, J=7.0 $\mathrm{Hz}, \operatorname{Ar~H}, 1 \mathrm{H}), 8.29(\mathrm{~d}, J=8.6 \mathrm{~Hz}, \mathrm{Ar} \mathrm{H}, 1 \mathrm{H}), 8.54(\mathrm{~d}, J=8.5 \mathrm{~Hz}, \mathrm{Ar} \mathrm{H}, 1 \mathrm{H})$. Synthesis of dKF-3. dKF-2 (15.2 $\mathrm{mg}, 0.0308 \mathrm{mmol}$ ) was dissolved in $1.0 \mathrm{~mL}$ of dry DMF. 1-(3-Dimethylaminopropyl)-3ethylcarbodiimide $(7.10 \mathrm{mg}, 0.0370 \mathrm{mmol})$ and 1-hydroxybenzotriazole monohydrate $(5.00 \mathrm{mg}$, $0.0370 \mathrm{mmol}$ ) suspended in $1.0 \mathrm{~mL}$ of dry DMF was added and the mixture was stirred for $30 \mathrm{~min}$, then $N$-dansyl pentenediamine $(16.7 \mathrm{mg}, 0.0499 \mathrm{mmol})$ dissolved in $1.0 \mathrm{~mL}$ of dry dichloromethane was added. The mixture was kept stirred for $15 \mathrm{~min}$. Ethyl acetate and $1 \mathrm{M}$ hydrochloric acid were added and the organic layer was separated. The water layer was extracted with ethyl acetate and the organic layers were combined, washed with brine, dried over anhydrous sodium sulfate, and evaporated under high vacuum. The resulting residue was purified by column chromatography $\left(\mathrm{CH}_{2} \mathrm{Cl}_{2}: \mathrm{MeOH}=9: 1\right)$, affording $15.7 \mathrm{mg}$ of yellow paste (63\% yield). dKF-3: ${ }^{1} \mathrm{H}$ NMR $\left(\mathrm{CDCl}_{3}\right.$ with $0.03 \%$ v/v TMS, $500 \mathrm{MHz}): \delta_{\mathrm{H}} 1.08-1.22\left(\mathrm{~m},-\mathrm{CH}_{2^{-}}, 2 \mathrm{H}\right), 1.26\left(\mathrm{~s},-\mathrm{C}\left(\mathrm{CH}_{3}\right)_{3}, 9 \mathrm{H}\right), 1.39\left(\mathrm{~s},-\mathrm{C}\left(\mathrm{CH}_{3}\right)_{3}, 9 \mathrm{H}\right), 1.26-$ $1.47\left(\mathrm{~m},-\mathrm{CH}_{2^{-}},-\mathrm{CH}_{2^{-}},-\mathrm{CH}_{2^{-}},-\mathrm{CH}_{2^{-}}, 8 \mathrm{H}\right), 1.58-1.62\left(\mathrm{~m},-\mathrm{CH}_{2^{-}}, 2 \mathrm{H}\right), 1.72-1.80\left(\mathrm{~m},-\mathrm{CH}_{2^{-}}, 2 \mathrm{H}\right), 2.89(\mathrm{~s},-$ $\left.\mathrm{N}\left(\mathrm{CH}_{3}\right)_{2}, 6 \mathrm{H}\right), 2.96-3.00\left(\mathrm{~m},-\mathrm{CH}_{2^{-}}, 2 \mathrm{H}\right), 3.06-3.11\left(\mathrm{~m},-\mathrm{CH}_{2^{-}}, 2 \mathrm{H}\right), 3.18-3.22\left(\mathrm{~m},-\mathrm{CH}_{2^{-}}, 2 \mathrm{H}\right), 4.61-4.65$ (m, -CH-, 1H), 5.08-5.10 (m, -CH-, 1H), 5.51 (brs, -NH-, 1H), 5.58 (brs, -NH-, 1H), 6.45 (brs, -NH-, 1H), 6.80 (brs, -NH-, 1H), 6.92 (brs, -NH-, 1H), 7.15-7.19 (m, Ar H, 5H), 7.50-7.58 (m, Ar H, 3H), 8.24 (d, $J=7.2 \mathrm{~Hz}, \operatorname{Ar} H, 1 \mathrm{H}), 8.35$ (d, J=7.2 Hz, Ar H, 1H), 8.53 (d, J=7.2 Hz, Ar H, 1H). Synthesis of dKF. dKF-3 (15.7 mg, $0.0194 \mathrm{mmol}$ ) was dissolved in $1.5 \mathrm{~mL}$ of dry dichloromethane, and trifluoroacetic acid (310 mg, $208 \mu \mathrm{L}, 2.72 \mathrm{mmol}$ ) was added. After stirring for $120 \mathrm{~min}$, the solvent was evaporated under vacuum. The resulting residue was purified by a high-performance liquid chromatography 
(HPLC, 1260, Agilent Technologies, Inc., California, USA) equipped with InertSustain C18 $5 \mu \mathrm{M}$ 20×150 mm (GL Sciences, Tokyo, Japan). The mobile phases were water containing $0.1 \%$ formic acid (A) and acetonitrile containing $0.1 \%$ formic acid (B), and the flow rate was set at $3.0 \mathrm{~mL} / \mathrm{min}$. The profile of the gradient is as follows: $20 \%$ B for $3 \mathrm{~min}, 20-40 \%$ B in $32 \mathrm{~min}, 40 \%$ B for $5 \mathrm{~min}, 40-20 \%$ B in $0.1 \mathrm{~min}$ followed by $20 \% \mathrm{~B}$ for another $4.9 \mathrm{~min}$ ( $45 \mathrm{~min}$ in total). The earned paste was washed with $1 \mathrm{M}$ hydrochloric acid and dried under vacuum, affording $6.1 \mathrm{mg}$ of a white amorphous solid (44\% yield, >99.5\% pure as assessed by HPLC/UV). dKF: ${ }^{1} \mathrm{H}$ NMR $\left(\mathrm{CD}_{3} \mathrm{OD}\right.$ with $0.05 \% \mathrm{v} / \mathrm{v} \mathrm{TMS}, 500$ $\mathrm{MHz}): \delta_{\mathrm{H}} 1.01-1.14\left(\mathrm{~m},-\mathrm{CH}_{2^{-}}, 2 \mathrm{H}\right), 1.19-1.30\left(\mathrm{~m},-\mathrm{CH}_{2^{-}}, 2 \mathrm{H}\right), 1.33-1.39\left(\mathrm{~m},-\mathrm{CH}_{2^{-}}, 2 \mathrm{H}\right), 1.41-1.48(\mathrm{~m},-$ $\left.\mathrm{CH}_{2^{-}}, 2 \mathrm{H}\right), 1.50-1.54\left(\mathrm{~m},-\mathrm{CH}_{2^{-}}, 2 \mathrm{H}\right), 1.69-1.76\left(\mathrm{~m},-\mathrm{CH}_{2^{-}}, 2 \mathrm{H}\right), 1.87-1.92\left(\mathrm{~m},-\mathrm{CH}_{2^{-}}, 2 \mathrm{H}\right), 2.82-2.89(\mathrm{~m}$, $\left.-\mathrm{CH}_{2^{-}}, 2 \mathrm{H}\right), 2.95-2.99\left(\mathrm{~m},-\mathrm{CH}_{2^{-}}, 2 \mathrm{H}\right), 3.01-3.07\left(\mathrm{~m},-\mathrm{CH}_{2^{-}}, 2 \mathrm{H}\right), 3.90(\mathrm{t}, \mathrm{J}=6.6 \mathrm{~Hz},-\mathrm{CH}-, 1 \mathrm{H}), 4.53(\mathrm{t}$, $J=7.5 \mathrm{~Hz},-\mathrm{CH}-, 1 \mathrm{H}), 4.86\left(\mathrm{~s},-\mathrm{N}\left(\mathrm{CH}_{3}\right)_{2}, 6 \mathrm{H}\right), 7.25-7.29(\mathrm{~m}, \mathrm{Ar} \mathrm{H}, 5 \mathrm{H})$, 7.85-7.92 (m, Ar H, 2H), 8.08 (d, $J=7.5 \mathrm{~Hz}, \operatorname{ArH}, 1 \mathrm{H}), 8.38$ (d, J=6.7 Hz, Ar H, 1H), 8.59 (d, J=8.6 Hz, Ar H, 1H), 8.93 (d, J=8.6 Hz, Ar H, $1 \mathrm{H})$.

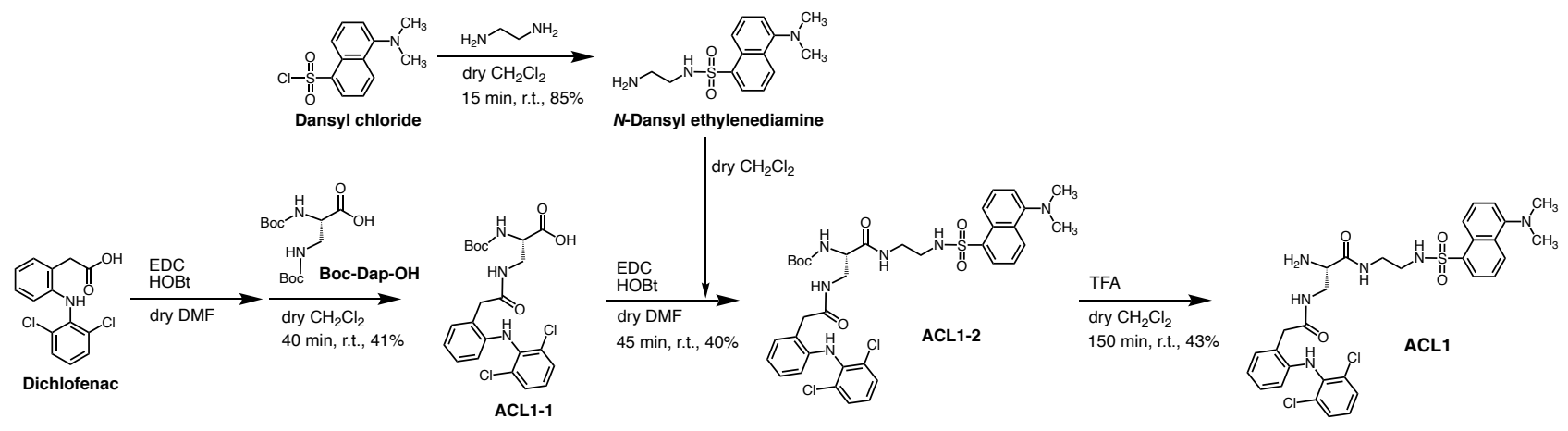

Scheme S1. Synthetic route for ACL1. 
Synthesis of ACL1. Synthesis of ACL1-1. Diclofenac (103 mg, $0.349 \mathrm{mmol}$ ) was dissolved in $1.0 \mathrm{~mL}$ of dry DMF. 1-(3-Dimethylaminopropyl)-3-ethylcarbodiimide $(81.4 \mathrm{mg}, 0.424 \mathrm{mmol})$ and 1hydroxybenzotriazole monohydrate $(58.6 \mathrm{mg}, 0.434 \mathrm{mmol})$ suspended in $1.0 \mathrm{~mL}$ of dry DMF was added and the mixture was stirred for $30 \mathrm{~min}$, then Boc-Dap-OH ( $35.7 \mathrm{mg}, 0.175 \mathrm{mmol}$ ) dissolved in $1.5 \mathrm{~mL}$ of dry dichloromethane was added. The mixture was kept stirred for another $10 \mathrm{~min}$. Ethyl acetate and $1 \mathrm{M}$ hydrochloric acid were added and the organic layer was separated. The water layer was extracted with ethyl acetate and the organic layers were combined, washed with brine, dried over anhydrous sodium sulfate, and evaporated under high vacuum. The resulting residue was purified by column chromatography $\left(\mathrm{CH}_{2} \mathrm{Cl}_{2}: \mathrm{MeOH}=9: 1\right)$, affording $34.8 \mathrm{mg}$ of colorless and transparent paste (41\% yield). ACL1-1: ${ }^{1} \mathrm{H}$ NMR (CD $\mathrm{CD}_{3} \mathrm{OD}$ with $\left.0.05 \% \mathrm{v} / \mathrm{v} \mathrm{TMS}, 500 \mathrm{MHz}\right): \delta_{\mathrm{H}} 1.41(\mathrm{~s},-$ $\left.\mathrm{C}\left(\mathrm{CH}_{3}\right)_{3}, 9 \mathrm{H}\right), 3.35\left(\mathrm{~s},-\mathrm{CH}_{2} \mathrm{O}-, 2 \mathrm{H}\right), 3.49-3.53\left(\mathrm{~m},-\mathrm{C}_{2} \mathrm{NH}-, 1 \mathrm{H}\right), 3.61-3.65\left(\mathrm{~m},-\underline{\mathrm{H}}_{2} \mathrm{NH}-, 1 \mathrm{H}\right), 4.22-4.23$ (m, $-\mathrm{CH}-, 1 \mathrm{H}), 6.38$ (dd, J=1.0, 8.0 Hz, Ar H, 1H), 6.87 (dt, J=1.1, 7.5 Hz, Ar H, 1H), 7.04 (dt, J=1.6, 7.6 $\mathrm{Hz}, \operatorname{Ar} \mathrm{H}, 1 \mathrm{H}), 7.06$ (t, J=8.1 Hz, Ar H, 1H), 7.51 (dd, J=1.1, 7.5 Hz, Ar H, 1H), 7.39 (d, J=8.1 Hz, Ar H, 2H). Synthesis of ACL1-2. ACL1-1 (31.1 mg, $0.0647 \mathrm{mmol}$ ) was dissolved in $1.0 \mathrm{~mL}$ of dry DMF where 1-(3-Dimethylaminopropyl)-3-ethylcarbodiimide $\quad\left(\begin{array}{lllll}15.0 & \mathrm{mg}, & 0.0781 & \mathrm{mmol}\end{array}\right)$ and 1 hydroxybenzotriazole monohydrate $(11.0 \mathrm{mg}, 0.0815 \mathrm{mmol})$ were suspended. The reaction mixture was stirred for $30 \mathrm{~min}$, then $\mathrm{N}$-dansyl ethylenediamine $(21.9 \mathrm{mg}, 0.0747 \mathrm{mmol}$ ) dissolved in $1.0 \mathrm{~mL}$ of dry dichloromethane was added. The mixture was kept stirred for 15 min. Ethyl acetate and 1M hydrochloric acid were added and the organic layer was separated. The water layer was extracted with ethyl acetate and the organic layers were combined, washed with brine, dried over anhydrous 
sodium sulfate, and evaporated under high vacuum. The resulting residue was purified by column chromatography $\left(\mathrm{CH}_{2} \mathrm{Cl}_{2}: \mathrm{MeOH}=99: 2\right)$, affording $19.7 \mathrm{mg}$ of yellow paste (40\% yield). ACL1-2: ${ }^{1} \mathrm{H}$ NMR $\left(\mathrm{CDCl}_{3}\right.$ with 0.03\% v/v TMS, $\left.500 \mathrm{MHz}\right): \delta_{\mathrm{H}} 1.42\left(\mathrm{~s},-\mathrm{C}\left(\mathrm{CH}_{3}\right)_{3}, 9 \mathrm{H}\right), 2.87\left(\mathrm{~s},-\mathrm{N}\left(\mathrm{CH}_{3}\right)_{2}, 6 \mathrm{H}\right), 2.98-$ $3.00\left(\mathrm{~m},-\underline{\mathrm{C}}_{2} \mathrm{NH}-, 2 \mathrm{H}\right), 3.18-3.25\left(\mathrm{~m},-\underline{\mathrm{C}}_{2} \mathrm{NH}-, 2 \mathrm{H}\right), 3.53-3.58\left(\mathrm{~m},-\mathrm{CHCH}_{2}-, 1 \mathrm{H}\right), 3.66-3.70(\mathrm{~m},-$

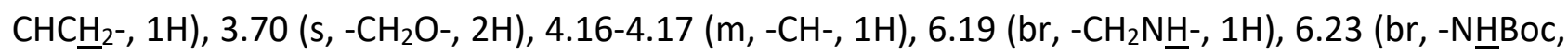
1H), 6.45 (d, J=8.0 Hz, Ar H, 1H), 6.83 (t, J=7.2 Hz, Ar H, 1H), 6.96-7.03 (m, - $\mathrm{CH}_{2} \mathrm{CON}^{-}-,-\mathrm{CON}^{-} \mathrm{HH}_{2^{-}}$, $\operatorname{Ar~H,~4H),~7.15-~7.16~(m,~Ar~H,~2H),~} 7.33(\mathrm{~d}, J=8.0 \mathrm{~Hz}, \operatorname{Ar} H, 2 H), 7.48(t, J=7.9 \mathrm{~Hz}, \operatorname{Ar~H,~1H),~} 7.54$ (t, $J=8.2 \mathrm{~Hz}, \operatorname{ArH}, 1 \mathrm{H}), 8.19(\mathrm{~d}, J=7.2 \mathrm{~Hz}, \operatorname{Ar~H}, 1 \mathrm{H}), 8.29(\mathrm{~d}, J=8.8 \mathrm{~Hz}, \operatorname{Ar} H, 1 \mathrm{H}), 8.52(\mathrm{~d}, J=8.5 \mathrm{~Hz}, \operatorname{Ar~H}$, 1H). Synthesis of ACL1. ACL1-2 (19.7 mg, $0.0261 \mathrm{mmol})$ was dissolved in $1.5 \mathrm{~mL}$ of dry dichloromethane, and trifluoroacetic acid (372 mg, $249 \mu \mathrm{L}, 3.26 \mathrm{mmol}$ ) was added. After stirring for $150 \mathrm{~min}$, the solvent was evaporated under vacuum. The resulting residue was purified by column chromatography $\left(\mathrm{CH}_{2} \mathrm{Cl}_{2}: \mathrm{MeOH}=95: 5\right)$, affording $7.3 \mathrm{mg}$ of yellow paste (43\% yield). ACL1: ${ }^{1} \mathrm{H}$ NMR $\left(\mathrm{CD}_{3} \mathrm{OD}\right.$ with $0.05 \%$ v/v TMS, $\left.500 \mathrm{MHz}\right): \delta_{\mathrm{H}} 2.90-3.00\left(\mathrm{~m},-\mathrm{C}_{2} \mathrm{NH}-, 2 \mathrm{H}\right), 3.04\left(\mathrm{~s},-\mathrm{N}\left(\mathrm{CH}_{3}\right)_{2}, 6 \mathrm{H}\right), 3.16-$ $3.20\left(\mathrm{~m},-\underline{\mathrm{H}}_{2} \mathrm{NH}-, 1 \mathrm{H}\right), 3.38-3.42\left(\mathrm{~m},-\underline{\mathrm{C}}_{2} \mathrm{NH}-, 1 \mathrm{H}\right), 3.57-3.61\left(\mathrm{~m},-\mathrm{CHC}_{2^{-}}, 1 \mathrm{H}\right), 3.68-3.71(\mathrm{~m},-$ $\left.\mathrm{CHCH}_{2}{ }^{-}, 1 \mathrm{H}\right), 3.72$ (d, J=14.2 Hz, - $\left.\underline{\mathrm{H}}_{2} \mathrm{CO}-, 1 \mathrm{H}\right), 3.77$ (d, J=14.1 Hz, - $\left.\underline{\mathrm{H}}_{2} \mathrm{CO}-, 1 \mathrm{H}\right), 3.98-3.99$ (m, -CH-, 1H), 6.34 (d, J=8.0 Hz, Ar H, 1H), $6.84(t, J=7.3 \mathrm{~Hz}, \operatorname{Ar~H}, 1 \mathrm{H}), 6.99$ (t, J=7.6 Hz, Ar H, 1H), 7.07 (t, J=7.7 $\mathrm{Hz}, \operatorname{Ar~H}, 1 \mathrm{H}), 7.21(\mathrm{~d}, J=7.4 \mathrm{~Hz}, \operatorname{Ar~H}, 1 \mathrm{H}), 7.39$ (d, J=8.1 Hz, Ar H, 2H), 7.49 (d, J=7.5 Hz, Ar H, 1H), 7.62-7.68 (m, Ar H, 2H), 8.22 (d, J=7.2 Hz, Ar H, 1H), 8.49 (d, J=8.7 Hz, Ar H, 1H), 8.53 (d, J=8.5 Hz, Ar $\mathrm{H}, 1 \mathrm{H}) .{ }^{13} \mathrm{C} \mathrm{NMR}\left(\mathrm{CD}_{3} \mathrm{OD}, 125 \mathrm{MHz}\right): \delta_{\mathrm{C}} 40.4,40.9,43.1,44.6,45.8(2 \mathrm{C}), 56.0,116.5,118.2,120.4$, $122.4,124.3,125.6,126.7,128.6,129.2,130.05$ (3C), 130.16, 130.9, 131.2, 131.3 (2C), 131.6, 136.8, 
139.2, 144.5, 153.3, 175.3, 175.6. HRMS-ESI $(m / z):[\mathrm{M}+\mathrm{Na}]^{+}$calcd for $\mathrm{C}_{31} \mathrm{H}_{34} \mathrm{Cl}_{2} \mathrm{~N}_{6} \mathrm{NaO}_{4} \mathrm{~S}, 679.1637$;

found, 679.1634 (-0.3 $\mathrm{mmu})$.

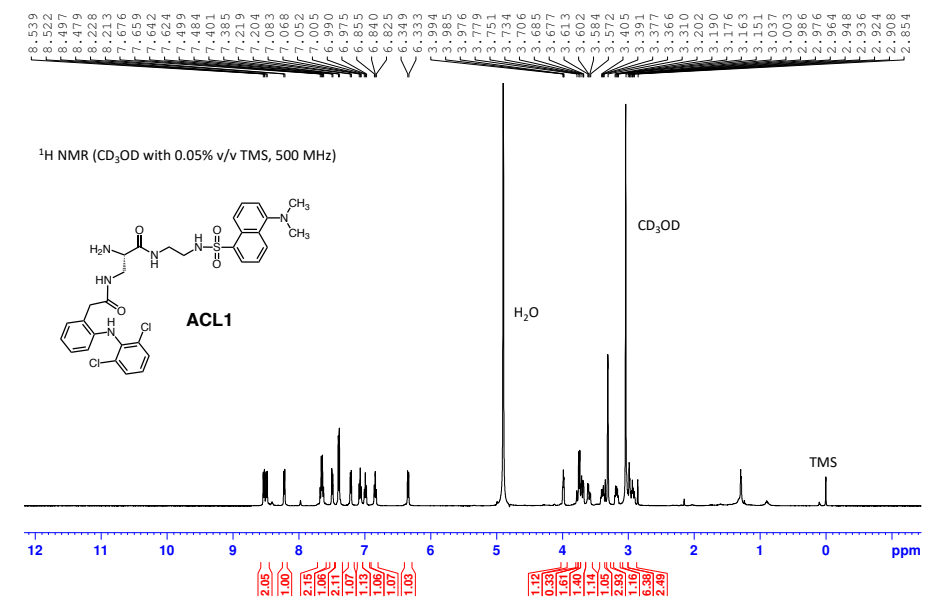

Figure S5. ${ }^{1} \mathrm{H}$ NMR spectrum of ACL1.

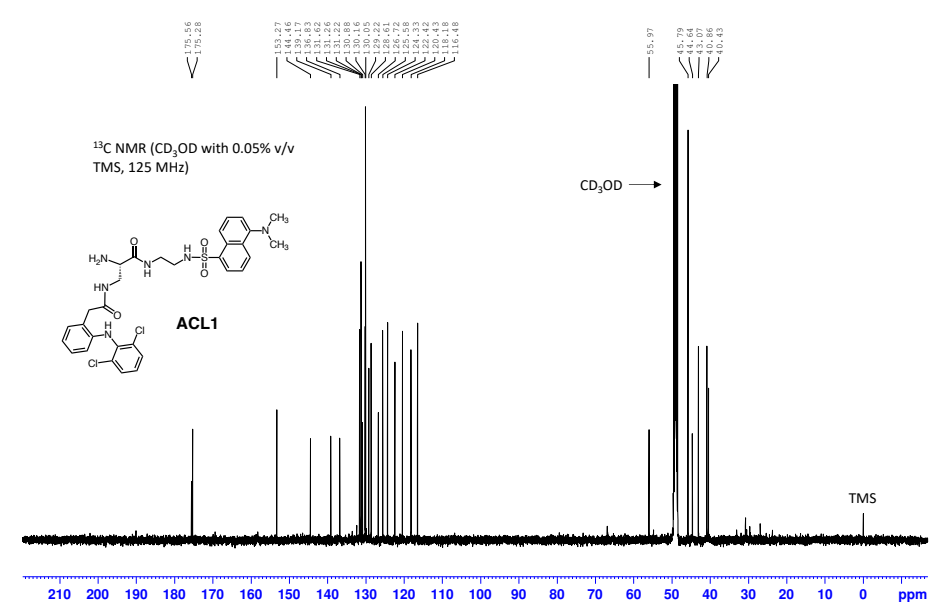

Figure S6. ${ }^{13} \mathrm{C}$ NMR spectrum of ACL1. 


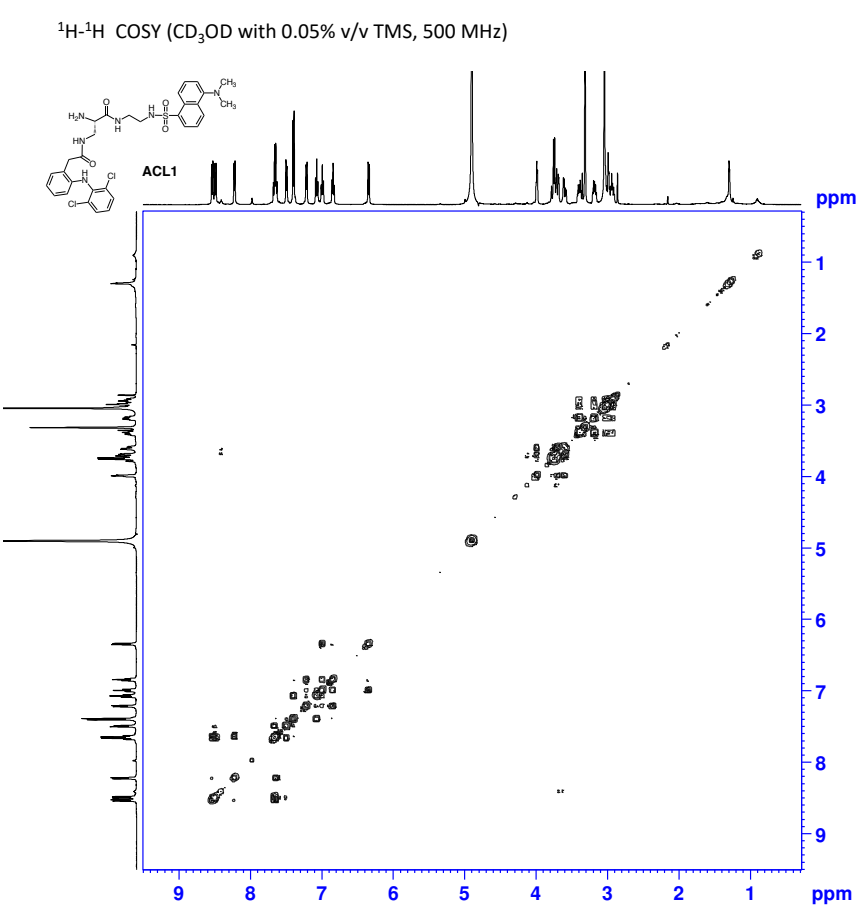

Figure S7. ${ }^{1} \mathrm{H}-{ }^{1} \mathrm{H}$ COSY spectrum of ACL1.

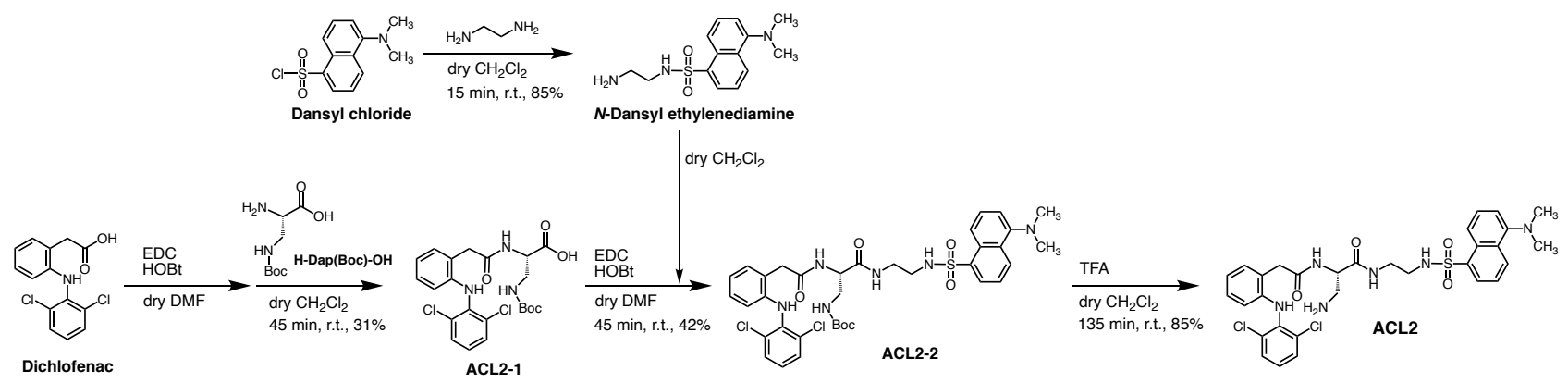

Scheme S4. Synthetic route for ACL2.

Synthesis of ACL2. Synthesis of ACL2-1. Diclofenac (101 mg, $0.342 \mathrm{mmol}$ ) was dissolved in $1.0 \mathrm{~mL}$ of dry DMF. 1-(3-Dimethylaminopropyl)-3-ethylcarbodiimide $(80.1 \mathrm{mg}, 0.417 \mathrm{mmol})$ and 1hydroxybenzotriazole monohydrate $(55.3 \mathrm{mg}, 0.410 \mathrm{mmol}$ ) suspended in $1.0 \mathrm{~mL}$ of dry DMF was added and the mixture was stirred for $30 \mathrm{~min}$, then $\mathrm{H}$-Dap(Boc)-OH (37.5 mg, $0.184 \mathrm{mmol}$ ) 
suspended in $1.0 \mathrm{~mL}$ of dry dichloromethane was added. The mixture was kept stirred for another 15 min. Ethyl acetate and $1 \mathrm{M}$ hydrochloric acid were added and the organic layer was separated. The water layer was extracted with ethyl acetate and the organic layers were combined, washed with brine, dried over anhydrous sodium sulfate, and evaporated under high vacuum. The resulting residue was purified by column chromatography $\left(\mathrm{CH}_{2} \mathrm{Cl}_{2}: \mathrm{MeOH}=95: 5 \rightarrow 10: 1\right)$, affording $27.1 \mathrm{mg}$ of colorless and transparent paste (31\% yield). ACL2-1: ${ }^{1} \mathrm{H} N M R\left(\mathrm{CD}_{3} \mathrm{OD}\right.$ with $\left.0.05 \% \mathrm{v} / \mathrm{v} \mathrm{TMS}, 500 \mathrm{MHz}\right)$ : $\delta_{H} 1.37\left(\mathrm{~s},-\mathrm{C}\left(\mathrm{CH}_{3}\right)_{3}, 9 \mathrm{H}\right), 3.40\left(\mathrm{dd}, J=7.0,13.3 \mathrm{~Hz},-\mathrm{CHC}_{2} 2^{-}, 1 \mathrm{H}\right), 3.51-3.54\left(\mathrm{~m}, \mathrm{CHC}_{2}{ }^{-}, 1 \mathrm{H}\right), 3.75(\mathrm{~s},-$ $\left.\mathrm{CH}_{2} \mathrm{CO}-, 2 \mathrm{H}\right), 4.38-4.40(\mathrm{~m},-\mathrm{CH}-, 1 \mathrm{H}), 6.37$ (d, J=7.6 Hz, Ar H, 1H), 6.86 (t, J=7.2 Hz, Ar H, 1H), 7.01$7.06(\mathrm{~m}, \operatorname{Ar~H}, 2 \mathrm{H}), 7.23(\mathrm{~d}, \mathrm{~J}=7.3 \mathrm{~Hz}, \mathrm{ArH}, 1 \mathrm{H}), 7.38$ (d, J=8.0 Hz, Ar H, 1H). Synthesis of ACL2-2. ACL21 (27.1 mg, $0.0563 \mathrm{mmol}$ ) was dissolved in $1.5 \mathrm{~mL}$ of dry DMF where 1-(3-Dimethylaminopropyl)-3ethylcarbodiimide $(17.3 \mathrm{mg}, 0.0901 \mathrm{mmol})$ and 1-hydroxybenzotriazole monohydrate $(10.6 \mathrm{mg}$, $0.0785 \mathrm{mmol}$ ) were suspended. The reaction mixture was stirred for $30 \mathrm{~min}$, then $\mathrm{N}$-dansyl ethylenediamine $(18.2 \mathrm{mg}, 0.0621 \mathrm{mmol})$ dissolved in $1.0 \mathrm{~mL}$ of dry dichloromethane was added. The mixture was kept stirred for 15 min. Ethyl acetate and $1 \mathrm{M}$ hydrochloric acid were added and the organic layer was separated. The water layer was extracted with ethyl acetate and the organic layers were combined, washed with brine, dried over anhydrous sodium sulfate, and evaporated under high vacuum. The resulting residue was purified by column chromatography $\left(\mathrm{CH}_{2} \mathrm{Cl}_{2}: \mathrm{MeOH}=99: 2\right)$, affording $18.1 \mathrm{mg}$ of yellow paste (42\% yield). ACL2-2: ${ }^{1} \mathrm{H} \mathrm{NMR}\left(\mathrm{CDCl}_{3}\right.$ with 0.03\% v/v TMS, $500 \mathrm{MHz}): \delta_{\mathrm{H}} 1.41\left(\mathrm{~s},-\mathrm{C}\left(\mathrm{CH}_{3}\right) 3,9 \mathrm{H}\right), 2.87\left(\mathrm{~s},-\mathrm{N}\left(\mathrm{CH}_{3}\right)_{2}, 6 \mathrm{H}\right), 2.96-2.99\left(\mathrm{~m},-\mathrm{C}_{2} \mathrm{NH}-\right.$, $2 \mathrm{H}), 3.12-3.14\left(\mathrm{~m},-\underline{\mathrm{C}}_{2} \mathrm{NH}-, 1 \mathrm{H}\right), 3.25-3.28\left(\mathrm{~m},-\mathrm{CH}_{2} \mathrm{NH}-, 1 \mathrm{H}\right), 3.44-3.46\left(\mathrm{~m},-\mathrm{CHCH}_{2}-, 2 \mathrm{H}\right), 3.75(\mathrm{~s},-$ 
$\left.\mathrm{C}_{2_{2}}, 1 \mathrm{H}\right), 4.21-4.23(\mathrm{~m},-\mathrm{CH}-, 1 \mathrm{H}), 5.14(\mathrm{t}, \mathrm{J}=6.0 \mathrm{~Hz},-\mathrm{NHBoc}, 1 \mathrm{H}), 5.92\left(\mathrm{t}, \mathrm{J}=5.5 \mathrm{~Hz},-\mathrm{CH}_{2} \mathrm{~N} \underline{\mathrm{H}}-, 1 \mathrm{H}\right), 6.47$ (t, J=8.1 Hz, Ar H, 1H), 6.80 (br, $\left.-\mathrm{CH}_{2} \mathrm{~N}_{\text {H}}-, 1 \mathrm{H}\right), 6.91$ (t, J=7.3 Hz, Ar H, 1H), 6.99 (t, J=8.1 Hz, Ar H, 1H), $7.08(t, J=7.7 \mathrm{~Hz}, \operatorname{Ar~H}, 1 \mathrm{H}), 7.15(\mathrm{~d}, J=7.5 \mathrm{~Hz}, \operatorname{Ar~H}, 1 \mathrm{H}), 7.20$ (d, J=8.4 Hz, Ar H, 1H), 7.33 (d, J=8.3 Hz, $\operatorname{Ar~H}, 2 \mathrm{H}), 7.42(\mathrm{br},-\mathrm{N} \underline{\mathrm{H}} \mathrm{CH}-, 1 \mathrm{H}), 7.47(\mathrm{t}, J=8.1 \mathrm{~Hz}, \operatorname{Ar~H}, 1 \mathrm{H}), 7.54(\mathrm{t}, J=8.0 \mathrm{~Hz}, \mathrm{Ar} \mathrm{H}, 1 \mathrm{H}), 8.18(\mathrm{~d}, J=7.3$ $\mathrm{Hz}, \operatorname{Ar~H}, 1 \mathrm{H}), 8.28(\mathrm{~d}, \mathrm{~J}=8.6 \mathrm{~Hz}, \mathrm{Ar} \mathrm{H}, 1 \mathrm{H}), 8.50(\mathrm{~d}, \mathrm{~J}=8.5 \mathrm{~Hz}, \mathrm{Ar} \mathrm{H}, 1 \mathrm{H})$. Synthesis of ACL2. ACL2-2 (18.1 $\mathrm{mg}, 0.0239 \mathrm{mmol}$ ) was dissolved in $3.0 \mathrm{~mL}$ of dry dichloromethane, and trifluoroacetic acid (423 mg, $284 \mu \mathrm{L}, 3.71 \mathrm{mmol}$ ) was added. After stirring for $135 \mathrm{~min}$, the solvent was evaporated under vacuum. The resulting residue was purified by column chromatography $\left(\mathrm{CH}_{2} \mathrm{Cl}_{2}: \mathrm{MeOH}=99: 2 \rightarrow 9: 1\right)$, affording $13.3 \mathrm{mg}$ of colorless and transparent paste (85\% yield). ACL2: ${ }^{1} \mathrm{H}$ NMR ( $\mathrm{CD}_{3} \mathrm{OD}$ with $0.05 \%$ v/v TMS, $500 \mathrm{MHz}): \delta_{\mathrm{H}} 2.86\left(\mathrm{~s},-\mathrm{N}\left(\mathrm{CH}_{3}\right)_{2}, 6 \mathrm{H}\right), 2.86-2.91\left(\mathrm{~m},-\mathrm{C}_{2} \mathrm{NH}-,-\mathrm{C}_{2} \mathrm{NH}_{2}, 3 \mathrm{H}\right), 3.00$ (dd, J=5.1 $\left.12.8 \mathrm{~Hz},-\underline{\mathrm{C}}_{2} \mathrm{NH}_{2}, 1 \mathrm{H}\right), 3.20-3.22\left(\mathrm{~m},-\underline{\mathrm{H}}_{2} \mathrm{NH}-, 1 \mathrm{H}\right), 3.74$ (d, J=14.4 Hz, $\left.-\underline{\mathrm{C}}_{2} \mathrm{CO}-, 1 \mathrm{H}\right), 3.81$ (d, J=14.1 $\left.\mathrm{Hz},-\underline{\mathrm{H}}_{2} \mathrm{CO}-, 1 \mathrm{H}\right), 4.32(\mathrm{t}, \mathrm{J}=5.7 \mathrm{~Hz},-\mathrm{CH}-, 1 \mathrm{H}), 6.31(\mathrm{t}, \mathrm{J}=7.8 \mathrm{~Hz}, \mathrm{Ar} \mathrm{H}, 1 \mathrm{H}), 6.85(\mathrm{t}, \mathrm{J}=7.3 \mathrm{~Hz}, \mathrm{Ar} \mathrm{H}, 1 \mathrm{H})$, $6.96(t, J=7.6 \mathrm{~Hz}, \operatorname{Ar~H}, 1 \mathrm{H}), 7.06(\mathrm{t}, J=7.8 \mathrm{~Hz}, \operatorname{Ar~H}, 1 \mathrm{H}), 7.24(\mathrm{t}, J=7.1 \mathrm{~Hz}, \operatorname{Ar~H}, 2 \mathrm{H}), 7.38$ (d, J=7.8 Hz, $\operatorname{Ar~H,~2H),~} 7.51(t, J=7.8 \mathrm{~Hz}, \operatorname{Ar~H}, 1 \mathrm{H}), 7.56(\mathrm{t}, J=8.0 \mathrm{~Hz}, \operatorname{Ar~H}, 1 \mathrm{H}), 8.14(\mathrm{~d}, J=7.2 \mathrm{~Hz}, \operatorname{Ar} \mathrm{H}, 1 \mathrm{H}), 8.30$ (d, J=8.6 Hz, Ar H, 1H), 8.52 (d, J=8.5 Hz, Ar H, 1H). ${ }^{13} \mathrm{C}$ NMR (CD $\left.{ }_{3} \mathrm{OD}, 125 \mathrm{MHz}\right): \delta_{c} 40.7$ (2C), 42.9, $44.1,45.8(2 \mathrm{C}), 57.0,116.5,118.3,120.4,122.5,124.3,125.6,126.6,128.7,129.2,130.05$ (3C), 130.11, 130.9, 131.20 (2C), 131.24, 131.7, 136.8, 139.2, 144.5, 153.2, 172.9, 174.9. HRMS-ESI (m/z): $[\mathrm{M}+\mathrm{Na}]^{+}$calcd for $\mathrm{C}_{31} \mathrm{H}_{34} \mathrm{Cl}_{2} \mathrm{~N}_{6} \mathrm{NaO}_{4} \mathrm{~S}, 679.1637$; found, 679.1634 (-0.3 mmu). 


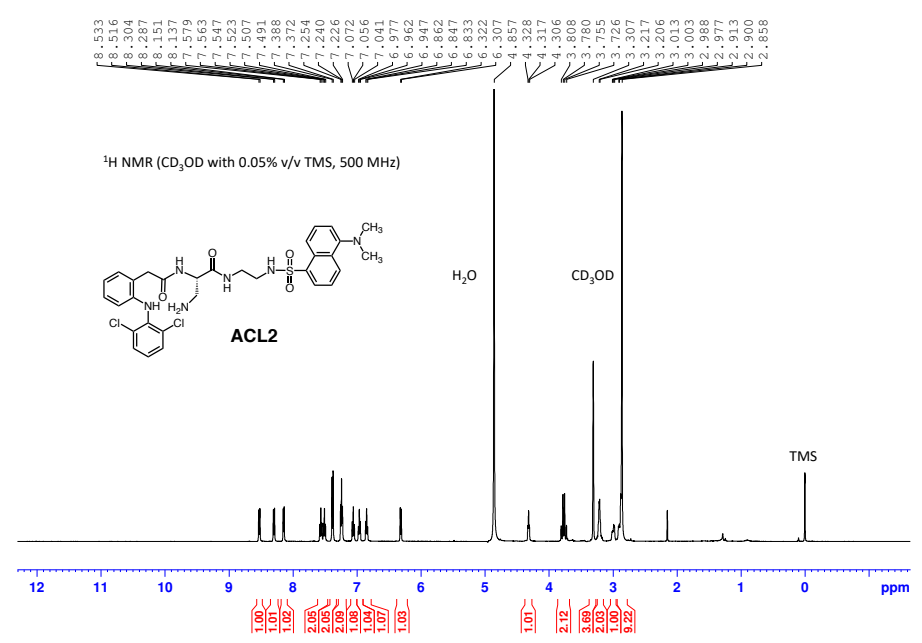

Figure S8. ${ }^{1} \mathrm{H}$ NMR spectrum of ACL2.

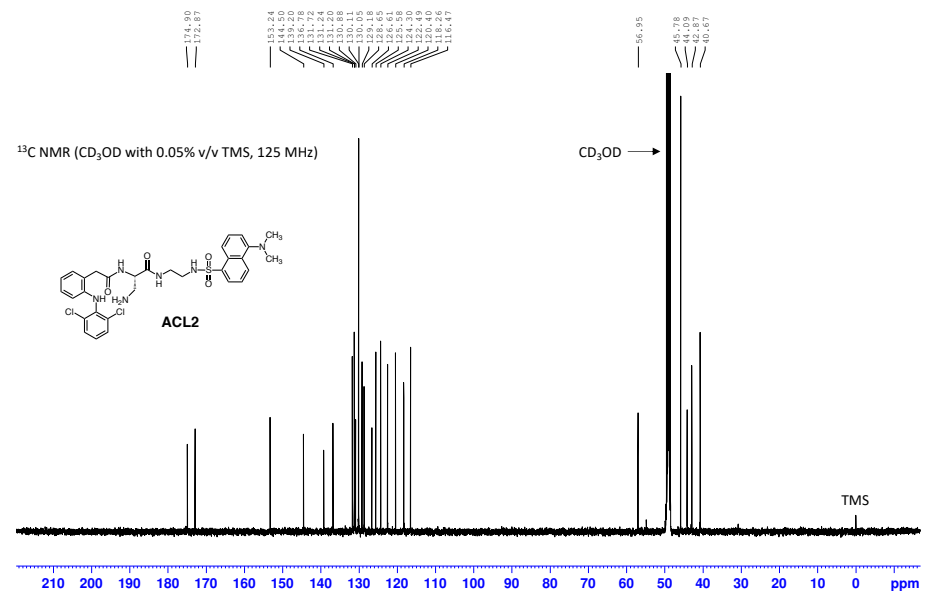

Figure S9. ${ }^{13} \mathrm{C}$ NMR spectrum of ACL2. 


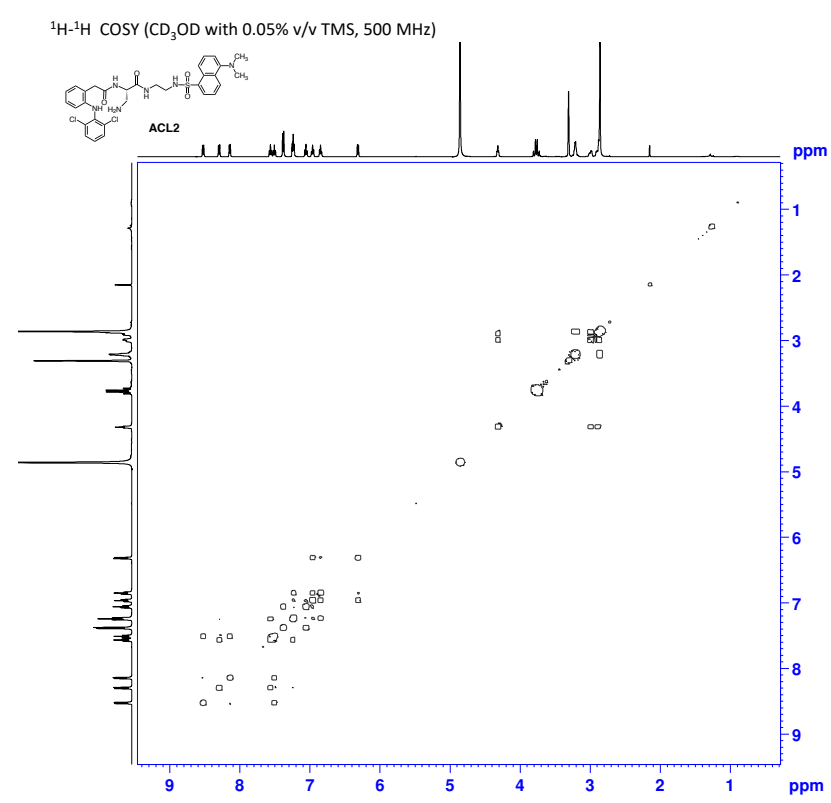

Figure $\mathrm{S} 10 .{ }^{1} \mathrm{H}-{ }^{1} \mathrm{H}$ COSY spectrum of $\mathrm{ACL2}$.

\section{Quantitative Analysis of Adduct Formation.}

The peaks detected only in the fluorescence chromatograms of complete samples were assumed as acylation or glycation adducts. Then, SIM analysis was performed to determine the type of adducts. The retention time for each adduct is shown in Table S1. To analyze adduct formation quantitatively, the adduct peak area in the fluorescence chromatograms was corrected by multiplying the correction constant $(f)$.

$$
\text { Area }_{\text {Corrected }}=\text { Area }_{\text {Detected }} \times f
$$

To determine $f$, we measured the fluorescence emission spectrum of each trapping reagent dissolved in various proportions of $A$ and $B$, the mobile phases of LC/MS. Each trapping reagent showed a linear increase in fluorescence emission intensity when the \% of B increased from 40 to $90 \%$ at an excitation wavelength of $340 \mathrm{~nm}$ (Figure S11). The fluorescence emission intensity was 
set at 1 when $B$ was $50 \% . p$ is the $\%$ of mobile phase (B) at the retention time when an adduct was detected.

$$
f_{\text {Dap-Dan }}=1 /(0.0237 p-0.2443), f_{\beta A l a-D a n}=1 /(0.0299 p-0.5151), f_{d K F}=1 /(0.0221 p-0.1192)
$$

Figure S11. Correlation between the \% of B and reciprocal of corrected constant $(f)$ for Dap-Dan,

$\beta A l a-D a n$ and dKF.
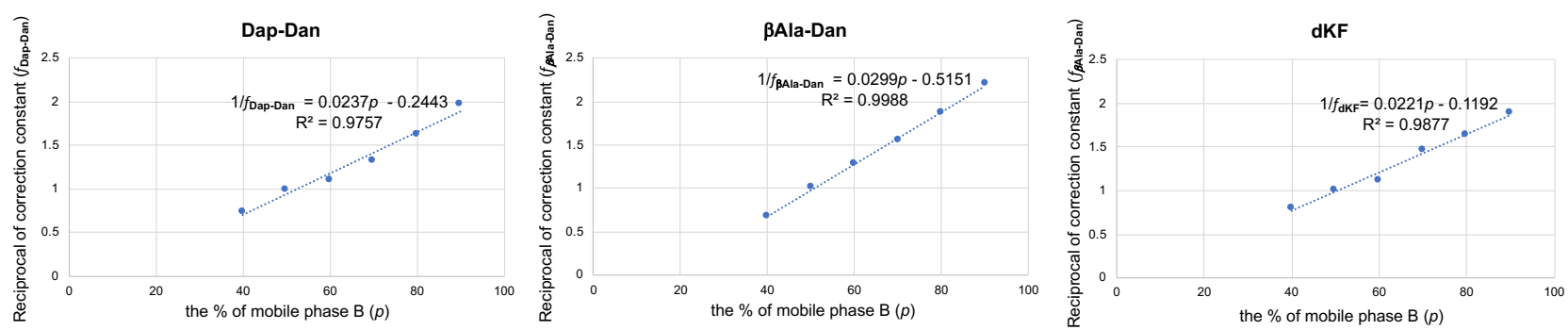
Table S1. Retention time for each adduct.

\begin{tabular}{|c|c|c|c|}
\hline & \multirow{2}{*}{ Drug } & \multicolumn{2}{|c|}{ Retention time [min] } \\
\hline & & Acylation & Glycation \\
\hline \multirow[t]{6}{*}{ Withdrawn } & Zomepirac & - & 19.4 \\
\hline & Bromfenac & - & 20.0 \\
\hline & Ibufenac & 23.2 & 23.7 \\
\hline & Benoxaprofen & 23.2 & 25.5 \\
\hline & Fenclofenac & 23.6 & 23.3 \\
\hline & & 23.9 & 24.6 \\
\hline \multirow[t]{10}{*}{ Warning } & Tolmetin & - & 18.0 \\
\hline & Diclofenac & 25.3 & 26.3 \\
\hline & & 25.9 & \\
\hline & Indomethacin & - & 19.2 \\
\hline & Ketoprofen & - & 18.1 \\
\hline & Fenoprofen & 19.2 & 20.8 \\
\hline & Ibuprofen & 25.0 & 24.2 \\
\hline & & & 24.5 \\
\hline & & & 24.6 \\
\hline & Mefenamic Acid & - & 19.7 \\
\hline \multirow[t]{5}{*}{ Safe } & Probenecid & - & - \\
\hline & Furosemide & - & - \\
\hline & Repaglinide & - & - \\
\hline & Gemfibrozil & - & - \\
\hline & Montelukast & - & - \\
\hline
\end{tabular}

$-:$ not detected 